PROCEEDINGS OF THE

AMERICAN MATHEMATICAL SOCIETY

Volume 130, Number 8, Pages 2425-2430

S 0002-9939(02)06317-7

Article electronically published on February 4, 2002

\title{
STONE'S DECOMPOSITION OF THE RENEWAL MEASURE VIA BANACH-ALGEBRAIC TECHNIQUES
}

\author{
M. S. SGIBNEV
}

(Communicated by Claudia M. Neuhauser)

\begin{abstract}
A Banach-algebraic approach to Stone's decomposition of the renewal measure is discussed. Estimates of the rate of convergence in a key renewal theorem are given.
\end{abstract}

\section{INTRODUCTION}

Let $F$ be a probability distribution on $\mathbf{R}$ with positive mean $\mu=\int_{\mathbf{R}} x F(d x)$, and let $H=\sum_{n=0}^{\infty} F^{n *}$ be the corresponding renewal measure; here $F^{1 *}:=F$, $F^{(n+1) *}:=F * F^{n *}, n \geq 1$, and $F^{0 *}:=\delta$, the atomic measure of unit mass at the origin. Suppose $F$ is spread-out, i.e., for some $m \geq 1, F^{m *}$ has a nonzero absolutely continuous component. Stone [14] showed that then there exists a decomposition $H=H_{1}+H_{2}$, where $H_{2}$ is a finite measure and $H_{1}$ is absolutely continuous with bounded continuous density $h(x)$ such that $\lim _{x \rightarrow \infty} h(x)=\mu^{-1}$ and $\lim _{x \rightarrow-\infty} h(x)=0$. Under additional assumptions, $h(x)$ has further nice properties [14, Theorem].

The measures $H_{1}$ and $H_{2}$ are constructed as follows. There exists an integer $p \geq 1$ such that $F^{p *}=F_{1}+F_{2}$, where $F_{1} \neq 0$ has a continuous density with compact support. Put $Q=\sum_{n=0}^{\infty} F_{2}^{p n *}$. Then $H_{1}:=F_{1} * Q * H$ and $H_{2}:=Q * \sum_{n=0}^{p-1} F^{n *}$ yield the desired decomposition [14] (see also [1, Theorem 2.6.2]).

In the present paper, we obtain Stone's decomposition $H=H_{1}+H_{2}$ by using Banach-algebraic techniques, which will allow us to extract detailed information about the asymptotic properties of the terms $H_{1}$ and $H_{2}$. We will show that, under suitable hypotheses, $H_{1}$ and $H_{2}$ belong to specific Banach algebras of measures. In this connection, it is appropriate to mention the paper by R. Grübel [4] as a major contribution to renewal theory based on Banach algebra techniques.

\section{Preliminaries}

Our discussion will rely on Banach algebras of measures with submultiplicative weights.

Received by the editors August 25, 2000 and, in revised form, February 19, 2001.

2000 Mathematics Subject Classification. Primary 60K05.

Key words and phrases. Stone's decomposition, renewal measure, asymptotic behavior, submultiplicative function, spread-out distribution, Banach algebra.

This research was supported by Grant 99-01-00504 of the Russian Foundation of Basic Research.

(C)2002 American Mathematical Society 
Definition 2.1. A function $\varphi(x), x \in \mathbf{R}$, is called submultiplicative if $\varphi(x)$ is a finite, positive, Borel-measurable function with the following properties:

$$
\varphi(0)=1, \quad \varphi(x+y) \leq \varphi(x) \varphi(y) \quad \forall x, y \in \mathbf{R} .
$$

We give some examples of such functions on $[0, \infty): \varphi(x)=(1+x)^{r}, r>0$; $\varphi(x)=\exp \left(c x^{\alpha}\right)$ with $c>0$ and $\alpha \in(0,1) ; \varphi(x)=\exp (r x)$ with $r \in \mathbf{R}$. Moreover, if $R(x), x \in \mathbf{R}_{+}$, is a positive, ultimately nondecreasing regularly varying function at infinity with a nonnegative exponent $\alpha$ (i.e., $R(t x) / R(x) \rightarrow t^{\alpha}$ for $t>0$ as $x \rightarrow \infty$ [3. Section VIII.8]), then there exist a nondecreasing submultiplicative function $\varphi(x)$ and a point $x_{0} \in(0, \infty)$ such that $c_{1} R(x) \leq \varphi(x) \leq c_{2} R(x)$ for all $x \geq x_{0}$, where $c_{1}$ and $c_{2}$ are some positive constants [9. Proposition]. The product of a finite number of submultiplicative functions is again a submultiplicative function.

It is well known [5, Section 7.6] that

$$
\begin{aligned}
-\infty<r_{1}:=\lim _{x \rightarrow-\infty} \frac{\log \varphi(x)}{x} & =\sup _{x<0} \frac{\log \varphi(x)}{x} \\
& \leq \inf _{x>0} \frac{\log \varphi(x)}{x}=\lim _{x \rightarrow \infty} \frac{\log \varphi(x)}{x}=: r_{2}<\infty
\end{aligned}
$$

and $M(h):=\sup _{|x| \leq h} \varphi(x)<\infty \forall h>0$.

Consider the collection $S(\varphi)$ of all complex-valued measures $\kappa$ such that $\|\kappa\|_{\varphi}:=$ $\int_{\mathbf{R}} \varphi(x)|\kappa|(d x)<\infty$; here $|\kappa|$ stands for the total variation of $\kappa$. The collection $S(\varphi)$ is a Banach algebra with norm $\|\cdot\|_{\varphi}$ by the usual operations of addition and scalar multiplication of measures, the product of two elements $\nu$ and $\kappa$ of $S(\varphi)$ is defined as their convolution $\nu * \kappa$ [5] Section 4.16]. The unit element of $S(\varphi)$ is the measure $\delta$. Define the Laplace transform of a measure $\kappa$ as $\widehat{\kappa}(s):=$ $\int_{\mathbf{R}} \exp (s x) \kappa(d x)$. Then relation (2.1) implies that the Laplace transform of any $\kappa \in S(\varphi)$ converges absolutely with respect to $|\kappa|$ for all $s$ in the strip $\Pi\left(r_{1}, r_{2}\right):=$ $\left\{s \in \mathbf{C}: r_{1} \leq \Re s \leq r_{2}\right\}$.

Let $\nu$ be a finite complex-valued measure. Denote by $T \nu$ the $\sigma$-finite measure with the density $v(x ; \nu):=\nu((x, \infty))$ for $x \geq 0$ and $v(x ; \nu):=-\nu((-\infty, x])$ for $x<0$. In the case $\int_{\mathbf{R}}|x||\nu|(d x)<\infty, T \nu$ is a finite measure whose Laplace transform is given by $(T \nu)^{\wedge}(s)=[\hat{\nu}(s)-\hat{\nu}(0)] / s, \Re s=0$, the value $(T \nu)^{\wedge}(0)$ being defined by continuity as $\int_{\mathbf{R}} x \nu(d x)<\infty$.

The absolutely continuous part of any distribution $F$ will be denoted by $F_{c}$, and its singular component by $F_{s}$, i.e., $F_{s}=F-F_{c}$.

\section{An ABSTRACT THEOREM}

Let $S\left(r_{1}, r_{2}\right)$ be the Banach algebra $S(\varphi)$ with $\varphi(x)=\max \left(e^{r_{1} x}, e^{r_{2} x}\right)$, where $r_{1} \leq 0 \leq r_{2}$. In this section, we shall consider Banach algebras $\mathcal{A}$ of measures such that (i) $\mathcal{A} \subset S\left(r_{1}, r_{2}\right)$ and (ii) each homomorphism $\mathcal{A} \mapsto \mathbf{C}$ is the restriction to $\mathcal{A}$ of some homomorphism $S\left(r_{1}, r_{2}\right) \mapsto$ C. Property (ii) can be restated as follows: Each maximal ideal $M$ of $\mathcal{A}$ is of the form $M_{1} \cap \mathcal{A}$, where $M_{1}$ is a maximal ideal of $S\left(r_{1}, r_{2}\right)$. It follows from the general theory of Banach algebras that if $\nu \in \mathcal{A}$ is invertible in $S\left(r_{1}, r_{2}\right)$, then $\nu^{-1} \in \mathcal{A}$.

In Sections 4 and 5 we apply the results of this section to the special case $\mathcal{A}=S(\varphi)$, where $\varphi(x)$ is an arbitrary submultiplicative function.

In what follows, $F$ will denote a spread-out probability distribution with finite mean $\mu>0$ such that $F \in S\left(r_{1}, r_{2}\right), r_{1} \leq 0 \leq r_{2} ;\left(F^{m *}\right)_{s}^{\wedge}\left(r_{i}\right)<1, i=1,2$, for 
some integer $m \geq 1$; and $\hat{F}(s) \neq 1 \forall s \in \Pi\left(r_{1}, r_{2}\right) \backslash\{0\}$. Let $L$ be the restriction of Lebesgue measure to $[0, \infty)$.

Theorem 3.1. Let $\mathcal{A}$ be a Banach algebra having properties (i) and (ii). Suppose $F, T F \in \mathcal{A}$. Then the renewal measure $H=\sum_{n=0}^{\infty} F^{n *}$ admits a Stone-type decomposition $H=H_{1}+H_{2}$, where $H_{2} \in \mathcal{A}$ and $H_{1}=L / \mu+r T H_{2}$ for some $r>r_{2}$. If, in addition, $T^{2} F \in \mathcal{A}$, then $H_{1}-L / \mu \in \mathcal{A}$.

Proof. Choose $r>r_{2}$. The function

$$
v(s):=\frac{(s-r)[1-\hat{F}(s)]}{s}, \quad s \in \Pi\left(r_{1}, r_{2}\right),
$$

is the Laplace transform of the measure $V:=\delta-F+r T F \in \mathcal{A}$, the value $v(0)$ being defined by continuity as $r \mu$. By Lemma 2 of [10] with obvious changes, there exists $W:=V^{-1} \in S\left(r_{1}, r_{2}\right)$, and hence $W \in \mathcal{A}$. We have $\hat{W}(s)=1 / v(s)$ and

$$
\begin{array}{r}
\hat{H}(s):=\frac{1}{1-\hat{F}(s)}=\frac{(s-r) \hat{W}(s)}{s}=\hat{W}(s)-\frac{r \hat{W}(0)}{s}-\frac{r[\hat{W}(s)-\hat{W}(0)]}{s} \\
=-\frac{1}{\mu s}+\hat{W}(s)-r(T W)^{\wedge}(s), \quad \Re s=0, s \neq 0 .
\end{array}
$$

Put $H_{2}:=W$ and $H_{1}:=L / \mu-r T W$. The desired decomposition follows from (3.1) [10, Lemmas 3 and 4]. (Note that in the case $F([0, \infty))=1$, relation (3.1) is the Laplace-transform version of the decomposition $H=H_{1}+H_{2}$.)

Finally, let $T^{2} F \in \mathcal{A}$. Then

$$
\begin{aligned}
(T W)^{\wedge}(s)= & \frac{\hat{W}(s)-\hat{W}(0)}{s}=-\frac{v(s)-v(0)}{s} \cdot \frac{1}{v(s) v(0)} \\
& =-\hat{W}(s)(T V)^{\wedge}(s) / v(0)=\hat{W}(s)\left[(T F)^{\wedge}(s)-r\left(T^{2} F\right)^{\wedge}(s)\right] / v(0) .
\end{aligned}
$$

This means that $T W=W *\left(T F-r T^{2} F\right) / v(0) \in \mathcal{A}$, and hence the second assertion of the theorem follows.

\section{Submultiplicative Case}

This section deals with submultiplicative moments of the measures $H_{1}-L / \mu$ and $H_{2}$ of the decomposition given by Theorem 3.1 .

Let $\varphi(x), x \in \mathbf{R}$, be a submultiplicative function such that $r_{1} \leq 0 \leq r_{2}$. By Theorem 1 of [7], $\mathcal{A}:=S(\varphi)$ satisfies properties (i) and (ii) of the preceding section, and hence Theorem 3.1 applies. We note some nuances. Relation $T F \in S(\varphi)$ implies $F \in S(\varphi)$. Actually,

$$
\begin{aligned}
\int_{0}^{\infty} \varphi(x) F((x, \infty)) d x & \geq \sum_{k=0}^{\infty} \inf _{x \in[k, k+1)} \varphi(x) F((k+1, k+2]) \\
& \geq \frac{1}{M(1)} \sum_{k=0}^{\infty} \int_{k+1}^{k+2} \varphi(x) F(d x)=\frac{1}{M(1)} \int_{1}^{\infty} \varphi(x) F(d x) .
\end{aligned}
$$

Since, obviously, $\int_{0}^{1} \varphi(x) F(d x)<\infty$, we have $\int_{0}^{\infty} \varphi(x) F(d x)<\infty$. Similarly, $\int_{-\infty}^{0} \varphi(x) F(d x)<\infty$. Therefore, instead of the hypotheses $F, T F \in S(\varphi)$ in Theorem 3.1, we may assume only $T F \in S(\varphi)$. Similarly, the set of conditions $F, T F$, $T^{2} F \in S(\varphi)$ may be replaced by $T^{2} F \in S(\varphi)$. Suppose now that $\varphi(x) / \exp \left(r_{1} x\right)$ 
is nonincreasing on $(-\infty, 0)$ and $\varphi(x) / \exp \left(r_{2} x\right)$ is nondecreasing on $[0, \infty)$. Theorem 3 of [11] implies that if $r_{1}=0=r_{2}$ and $\int_{\mathbf{R}}(1+|x|)^{k} \varphi(x) F(d x)<\infty$ for some integer $k \geq 1$, or if $r_{1}<0=r_{2}$ and $\int_{0}^{\infty}(1+x)^{k} \varphi(x) F(d x)<\infty$, or if $r_{1}=0<r_{2}$ and $\int_{-\infty}^{0}(1+|x|)^{k} \varphi(x) F(d x)<\infty$, then $T^{k} F \in S(\varphi)$. If $r_{1}<0<r_{2}$, then $F \in S(\varphi) \Rightarrow T^{k} F \in S(\varphi) \forall k \geq 1$ [11 Theorem 2]. Suppose now that $r_{1}=0=r_{2}$. Then, instead of the hypotheses $F, T F \in S(\varphi)$ in Theorem 3.1 we may assume only $F \in S\left(\varphi_{1}\right)$, where $\varphi_{1}(x):=(1+|x|) \varphi(x)$. Similarly, the set of conditions $F$, $T F, T^{2} F \in S(\varphi)$ may be replaced by $F \in S\left(\varphi_{2}\right)$, where $\varphi_{2}(x):=(1+|x|)^{2} \varphi(x)$. In the latter case, $H_{2}$ will be in $S\left(\varphi_{1}\right)$. Suppose $r_{1}<0<r_{2}$. Then the set of conditions $F, T F, T^{2} F \in S(\varphi)$ may be replaced by $F \in S(\varphi)$. The intermediary cases $r_{1}<0=r_{2}$ and $r_{1}=0<r_{2}$ are dealt with in a similar way.

Corollary 4.1. Let $\varphi(x)$ be a submultiplicative function such that $r_{1} \leq 0 \leq r_{2}$. Suppose that $\varphi(x)$ is nonincreasing on $(-\infty, 0)$ and nondecreasing on $[0, \infty)$. Assume $T F \in S(\varphi)$. Fix any $h>0$. Then

$$
|H-L / \mu|((x, x+h])=o(1 / \varphi(x)) \quad \text { as }|x| \rightarrow \infty .
$$

Proof. For the sake of definiteness, we consider the case $x \rightarrow \infty$. By Theorem 1, $H_{2} \in S(\varphi)$, and hence

$$
\begin{gathered}
\varphi(x)|H-L / \mu|((x, x+h]) \leq \int_{x}^{x+h} \varphi(y)\left|H_{2}\right|(d y)+r \int_{x}^{x+h} \varphi(y)\left|H_{2}((y, \infty))\right| d y \\
\leq \int_{x}^{\infty} \varphi(y)\left|H_{2}\right|(d y)+r h M(h) \varphi(x)\left|H_{2}\right|((x, \infty)) \\
\quad \leq[1+r h M(h)] \int_{x}^{\infty} \varphi(y)\left|H_{2}\right|(d y)=o(1) \quad \text { as } x \rightarrow \infty
\end{gathered}
$$

Remark 4.2. In the case $r_{1}=r_{2}=0$, the assertion of Corollary 4.1 was obtained in 6, Corollary 3] (see also [1, Theorem 2.6.4 (b)], where $\varphi(x) \equiv 1$ ).

\section{Convergence Rates in a key Renewal theorem}

Consider the renewal equation

$$
X(t)=g(t)+\int_{\mathbf{R}} X(t-y) F(d y)=: g(t)+X * F(t),
$$

where $g \in L_{1}(\mathbf{R})$ and $F$ is a spread-out probability distribution on $\mathbf{R}$ with positive mean $\mu$. The function $X(t):=g * H(t)+c$ is clearly a solution to (5.1); here $c$ is any constant. So the asymptotic properties of the solution to (5.1) are those of the convolution $g * H(t)$, which, under various assumptions, have been studied by several authors [12, 13, 8, 2, 1]. Some properties of $X(t)$ were later rediscovered in a slightly more general setting [15, Theorem 5.1]. Usually, assertions about the asymptotic behavior of $g * H(t)$ are called key renewal theorems. As pointed out in [2] and carried out in [1], Stone's decomposition allows us to obtain an elegant proof of a key renewal theorem, which in simplified form can be stated as follows:

$$
g * H(t) \rightarrow \begin{cases}\mu^{-1} \int_{\mathbf{R}} g(x) d x & \text { as } t \rightarrow \infty \\ 0 & \text { as } t \rightarrow-\infty\end{cases}
$$

provided that $g(x)$ is bounded and $\lim _{|x| \rightarrow \infty} g(x)=0$. 
In this section, we shall obtain submultiplicative rates of convergence in (5.2) by means of the Stone-type decomposition of Theorem 3.1 with $\mathcal{A}=S(\varphi)$.

Theorem 5.1. Let $\varphi(x)$ be a submultiplicative function such that $r_{1} \leq 0 \leq r_{2}$, and let $g(x), x \in \mathbf{R}$, be a Borel-measurable function such that $(a) g \in L_{1}(\mathbf{R})$, (b) $g \cdot \varphi \in L_{\infty}(\mathbf{R}),(c) g(x) \varphi(x) \rightarrow 0$ as $|x| \rightarrow \infty$ outside a set of Lebesgue measure zero, and $(d) \varphi(t) \int_{t}^{\infty}|g(x)| d x \rightarrow 0$ as $t \rightarrow \infty$ and $\varphi(t) \int_{-\infty}^{t}|g(x)| d x \rightarrow 0$ as $t \rightarrow-\infty$. Suppose $T^{2} F \in S(\varphi)$. Then, as $t$ approaches $\pm \infty$ outside a set of Lebesgue measure zero,

$$
\sup _{\alpha:|\alpha| \leq|g|}\left|\alpha * H(t)-\mu^{-1} \int_{\mathbf{R}} \alpha(x) d x\right|=o\left(\frac{1}{\varphi(t)}\right)
$$

and $\sup _{\alpha:|\alpha| \leq|g|}|\alpha * H(t)|=o(1 / \varphi(t))$, the $\alpha(x)$ being Borel-measurable functions on $\mathbf{R}$.

Proof. By Theorem 3.1 with $\mathcal{A}=S(\varphi)$, both $H_{1}-L / \mu$ and $H_{2}$ are elements of $S(\varphi)$. Choose $\tilde{g} \in L_{1}(\mathbf{R})$ such that $\tilde{g}=g$ a.e., $\sup _{x \in \mathbf{R}}|\tilde{g}(x)| \varphi(x)<\infty$, and $\tilde{g}(x) \varphi(x) \rightarrow 0$ as $|x| \rightarrow \infty$ in the usual sense. It suffices to put $\tilde{g}(x)=0$ on $\left\{x \in \mathbf{R}:|g(x)| \varphi(x)>\|g \cdot \varphi\|_{\infty}\right\}$ and on a set, say $B$, of Lebesgue measure zero such that $\lim _{x \notin B,|x| \rightarrow \infty} g(x) \varphi(x)=0$; otherwise, $\tilde{g}(x):=g(x)$. By Fubini's theorem, the sets $A_{1}:=\left\{x:|\tilde{g}| *\left|H_{1}-L / \mu\right|(x) \neq|g| *\left|H_{1}-L / \mu\right|(x)\right\}$ and $A_{2}:=\left\{x:|\tilde{g}| *\left|H_{2}\right|(x) \neq\right.$ $\left.|g| *\left|H_{2}\right|(x)\right\}$ are both of Lebesgue measure zero. Set $A:=A_{1} \cup A_{2}$. We have

$$
\varphi(t)|\tilde{g}| *\left|H_{2}\right|(t) \leq \int_{\mathbf{R}}|\tilde{g}(t-x)| \varphi(t-x) \varphi(x)\left|H_{2}\right|(d x) .
$$

By dominated convergence, it follows from the hypotheses of the theorem that the right-hand side tends to zero as $t \rightarrow \infty$, and so does the left-hand side. By the same reasons, $\lim _{t \rightarrow \infty} \varphi(t)|\tilde{g}| *\left|H_{1}-L / \mu\right|(t)=0$. Hence both $\varphi(t)|g| *\left|H_{1}-L / \mu\right|(t)$ and $\varphi(t)|g| *\left|H_{2}\right|(t)$ tend to zero as $t \rightarrow \infty$, remaining outside the set $A$ of Lebesgue measure zero. The first assertion of the theorem now follows from the obvious inequality

$$
\begin{aligned}
\left|\alpha * H(t)-\mu^{-1} \int_{\mathbf{R}} \alpha(x) d x\right| \leq & |g| *\left|H_{1}-L / \mu\right|(t)+|g| *\left|H_{2}\right|(t) \\
& +\mu^{-1} \int_{t}^{\infty}|g(x)| d x
\end{aligned}
$$

and condition $(\mathrm{d})$. The case $t \rightarrow-\infty$ is dealt with in a similar way.

\section{REFERENCES}

1. G. Alsmeyer, Erneuerungstheorie, B.G. Teubner, Stuttgart, 1991. MR 92f:60148

2. E. Arjas, E. Nummelin, R. L. Tweedie, Uniform limit theorems for non-singular renewal and Markov renewal processes. J. Appl. Probab. 15 (1978), 112-125. MR 57:7798

3. W. Feller, An Introduction to Probability Theory and Its Applications II, Wiley, New York, 1966. MR 35:1048

4. R. Grübel, On subordinated distributions and generalized renewal measures. Ann. Probab. 15 (1987), 394-415. MR 88d:60045

5. E. Hille, R. S. Phillips, Functional Analysis and Semi-Groups, Amer. Math. Soc. Colloquium Publications, vol. 31, Providence, RI, 1957. MR 19:664d

6. B. A. Rogozin, Asymptotics of renewal functions. Theory Probab. Appl. 21 (1976), 669-686. MR 54:8911

7. B. A. Rogozin, M. S. Sgibnev, Banach algebras of measures on the line. Siberian Math. J. 21 (1980), 265-273. MR 81e:43003 
8. M. Schäl, Über Lösungen einer Erneuerungsgleichung. Abh. Math. Sem. Univ. Hamburg 36 (1971), 89-98. MR 48:12659

9. M. S. Sgibnev, Submultiplicative moments of the supremum of a random walk with negative drift. Statist. Probab. Lett. 32 (1997), 377-383. MR 99e:60158

10. M. S. Sgibnev, Exact asymptotic behaviour in a renewal theorem for convolution equivalent distributions with exponential tails. SUT Journal of Mathematics 35 (1999), 247-262. MR 2000m:60102

11. M. S. Sgibnev, An asymptotic expansion for the distribution of the supremum of a random walk. Studia Math. 140 (2000), 41-55. MR 2001g:60109

12. W. L. Smith, Regenerative stochastic processes. Proc. R. Soc. London A 232 (1955), 6-31. MR 17:502b

13. W. L. Smith, Remarks on the paper 'Regenerative stochastic processes'. Proc. R. Soc. London A 256 (1960), 496-501. MR 22:6025

14. C. Stone, On absolutely continuous components and renewal theory. Ann. Math. Statist. 37 (1966), 271-275. MR 33:4981

15. N. B. Yengibarian, Renewal equation on the whole line. Stochastic Process. Appl. 85 (2000), 237-247. MR 2001d:60097b

Institute of Mathematics, Siberian Branch of the Russian Academy of Sciences, Novosibirsk 90, 630090 RUSSIA

E-mail address: sgibnev@math.nsc.ru 